\title{
Trivium
}

Revue franco-allemande de sciences humaines et sociales - Deutsch-französische Zeitschrift für Geistesund Sozialwissenschaften

$20 \mid 2015$

Réflexivité et Système. Le débat sur l'ordre et l'autoorganisation dans les années 1970

\section{Das Order from noise-Prinzip, das nicht-direktive Lernen und der Traum}

\section{Henri Atlan}

Traducteur : Andreas Pfeuffer

\section{OpenEdition \\ Journals}

Édition électronique

URL : http://journals.openedition.org/trivium/5198

ISSN : 1963-1820

Éditeur

Les éditions de la Maison des sciences de l'Homme

\section{Référence électronique}

Henri Atlan, « Das Order from noise-Prinzip, das nicht-direktive Lernen und der Traum », Trivium [Online], 20 | 2015, online erschienen am 16 Juni 2015, abgerufen am 08 September 2020. URL : http:// journals.openedition.org/trivium/5198

Ce document a été généré automatiquement le 8 septembre 2020

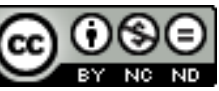

Les contenus des la revue Trivium sont mis à disposition selon les termes de la Licence Creative Commons Attribution - Pas d'Utilisation Commerciale - Pas de Modification 4.0 International. 


\title{
Das Order from noise-Prinzip, das nicht-direktive Lernen und der Traum
}

\author{
Henri Atlan
}

Traduction : Andreas Pfeuffer

\section{NOTE DE L'ÉDITEUR}

Wir danken Herrn Henri Atlan für die freundliche Genehmigung, diesen Artikel in deutscher Übersetzung zu publizieren.

Nous remercions M. Henri Atlan de nous avoir accordé l'autorisation de traduire ce texte pour le présent numéro.

$1 \mathrm{Zu}$ den Schwierigkeiten, die sich einstellen, will man Brücken zwischen der Biologie und der Anthropologie schlagen, zählt jene, die logischen Vorannahmen zu erkennen. Diese logischen Vorannahmen fördern nämlich inadäquate Auffassungen zutage, die, wie ich glaube, aus dem Fehlen einer Theorie der Komplexität bzw. der Organisation herrühren. Von Neumann hat, insbesondere in seinen letzten Lebensjahren, die Notwendigkeit solcher Theorien betont, die mit zunehmendem Komplexitätsgrad der untersuchten Systeme noch drängender wird, selbst wenn man sich darüber noch nicht im Klaren ist, wie die Komplexität zu messen ist. Je komplexer sich das untersuchte System intuitiv und noch auf sehr vage Weise darstellt, desto stärker beeinflusst die Art und Weise, wie man die Frage formuliert und an das System heranträgt, die Schlüsse, die man aus denselben Beobachtungen zieht. Ich möchte das anhand der Experimente von Jacobson zum Aufbau der Sehnerven illustrieren. Wie man uns erklärt ${ }^{1}$, strecken die retinalen Neuronen ihre Fortsätze auf genau festgelegte Weise in bestimmte Regionen zunächst des corpus geniculatum (Kniehöcker), dann des Okzipitalcortex. Dieser strenge Determinismus ist dermaßen ausgeprägt, dass er sich auch in einer räumlichen Anordnung niederschlägt. So strecken nicht nur die retinalen Neuronen ihre Fortsätze in eine bestimmte Region des Cortex, sondern die Neuronen, die sich 
beispielsweise im äußeren Teil der Retina befinden, strecken die Ihren in einen bestimmten Teil des Cortex, diejenigen, die sich unten befinden, in einen anderen Teil. Jacobson hatte nun die Idee, Transplantationen vorzunehmen, also beispielsweise eine rechte Retinaanlage nach links zu versetzen oder sie durch die Versetzung des oberen Teils nach unten umzukehren, um dann zu sehen, ob die Nervenfasern auch in die gleiche Richtung drängen oder umgelenkt werden. Es zeigte sich, dass dies vom Entwicklungsstadium abhing, in dem diese Transplantation vorgenommen wurde. Geschieht die Transplantation früh in der Entwicklung, ist die Retinaanlage gewissermaßen empfänglich für das, was Wolpert eine »Positionsinformation « genannt hat. Es sieht ganz so aus, als ob die Neuronen ein Gespür dafür hätten, ob sie sich rechts oder links, oben oder unten befinden, und imstande wären, diese Information den benachbarten Neuronen zu übermitteln. Und wenn nach Ablauf einer bestimmten Zeit die Transplantation zu spät vorgenommen wird, sieht es so aus, als ob diese Neuronen diese Positionsinformation nicht mehr wahrnehmen würden. Niemand kennt gegenwärtig den physikalischen Träger dieser Positionsinformation. Vorstellbar ist, dass es Konzentrationsgradienten von chemischen Substanzen sind, letztlich hat aber noch niemand den Beweis für diese Hypothese erbracht. Denkbar ist auch die Übertragung von Informationsmolekülen von Zelle zu Zelle, und es gibt auch schon elektronenmikroskopische Dokumente, die zeigen, dass sich im Zuge der Entwicklung Botenstoffe scheinbar verringern und verschwinden. Das legt den Gedanken nahe, dass die Spezifizierung sich in Form einer Verringerung der Informationsübertragung zwischen den Neuronen vollzieht, d.h. durch eine Art Hemmung in dieser Informationsübertragung, die in die Fixierung eines pattern mündet. Betrachtet man diese Experimente unter diesem Gesichtspunkt, dann gelangt man zu Schlüssen, die sich von denjenigen unterscheiden, die besagen: »Die Entwicklung vollzieht sich strikt ohne Einfluss der Umwelt; der Beweis hierfür ist, dass sich die Entwicklung unabhängig davon vollzieht, ob die Netzhaut durch Lichtflimmern stimuliert wird oder nicht.« In der Tat betrachtet man in letzterem Fall die Wechselwirkungen mit der Umwelt nur in Gestalt von Lichtstimuli, während es im erstgenannten Fall mit der Umwelt eine Wechselwirkung in Gestalt von Positionsinformationen gibt, und nicht in Gestalt von Lichtreizen. Man weiß noch nicht genau, woraus sie bestehen, doch sie stellen zweifellos etwas dar, jedenfalls aufgrund ihrer Wirkungen.

2 Eine weitere Schwierigkeit: die Frage nach genetischen Programmen als dem Ursprung von Verhaltensweisen. Man sagt, wenn man zwei identische Verhaltenspatterns beobachtet, impliziere das den Gesetzen der Logik zufolge, dass ein identischer Erzeugungsmechanismus für diese patterns vorgelegen haben muss. Ich glaube, dass das vollkommen falsch ist, da diese formale Identität zwischen zwei patterns nur vom Standpunkt des Betrachters aus eine ist, dass sie also die dem Betrachter eigenen Mechanismen des Formenerkennens ins Spiel bringt. Diese Mechanismen des Formenerkennens sind aber äußerst kompliziert. Nehmen wir ein Beispiel.

Wenn man zunächst einen Kreis betrachtet, dann einen gepunkteten Kreis, dann wird man, wenn die Pünktchen weiter auseinanderrücken, nur bis $z u$ einem gewissen Zwischenraum noch erkennen, dass es sich um einen Kreis handelt. Ab welchem Moment wird man keinen Kreis mehr erkennen? Der Übergang ist offenbar ein gleitender und hängt von den uns eigenen Mechanismen der Formerkennung ab. Darüber hinaus wirft der Begriff der Programme, insbesondere von genetischen Programmen, eine ganze Reihe von Problemen auf. Ich glaube, dass hierin ein Begriff 
inkorrekt gebraucht wird, der tatsächlich eine Metapher darstellt. Der Programmbegriff ist direkt aus der Computersprache abgeleitet, und in diesem Fall wird das Programm von außerhalb der Maschine geliefert. Im Fall des genetischen Programms gibt es zwei Möglichkeiten: Entweder sagt man, dass das genetische Programm in der DNA enthalten ist. Dann kann man offenbar davon sprechen, dass die DNA von außerhalb der Maschine geliefert wird, weil sie von den Eltern stammt. Doch kann man sich fragen, inwiefern es legitim ist, die DNA wirklich als ein Programm anzusehen, weil nicht so sehr die Folge der Gene von Bedeutung ist als vielmehr die Mechanismen der Genregulation, d.h. der Regulation der Genexpression. Diese Mechanismen sorgen nämlich dafür, dass neben der DNA die zahlreichen Proteine als Initiations- und Terminationsfaktoren ebenso wie die Repressoren, die RNAPolymerasen usw. zum Einsatz kommen. So gelangt man zu der schon von zahlreichen anderen aufgezeigten Schwierigkeit, dass man nämlich sagt, es handele sich um ein eigenartiges Programm, da es zu seiner Ausführung die Produkte seines eigenen Lesens und seiner eigenen Ausführung benötigt. Letztlich nimmt man an, dass das Programm nicht aus der DNA allein, sondern ganz umfassend aus der gesamten Genregulationsmaschinerie besteht. Das Programm koinzidiert also mit beinahe der Gesamtheit der Zelle, und so kommt man auch zu dem häufig verwendeten Begriff der endogenen Programme. Abgesehen von diesen sind jedoch noch nie jemandem solche endogenen Programme begegnet, da eben der Begriff Programm aus der Computersprache kommt, wo die Programme letzten Endes immer von außen kommen. Aus diesem Grund war ich ganz glücklich darüber, dass Jacques Monod, als er vom genetischen Code sprach, sagte, dass es sich tatsächlich um eine Replikationsmaschinerie nach Art eines - wenn sie so wollen - Fotokopierers handele. ${ }^{2}$ Man sollte in der Tat, wenn man von einem genetischen Code spricht, lieber nur von einem genetischen Speicher sprechen, von einem Speicher immer noch im Sinne der Computertechnologie, das heißt, einem Mechanismus der Replikation, schlicht wie bei einem Magnetband, auf dem die Informationen gespeichert sind, das aber absolut keinen Einfluss darauf hat, wie diese Information anschließend genutzt wird. Hat man sich diese Schwierigkeiten erst klar gemacht, stellt sich die Frage: Wie können diese Systeme funktionieren, wie sind sie organisiert? Daher hat sich eine Reihe von Forschern mit sogenannten Selbstorganisationsmechanismen auseinandergesetzt. Die Methoden, mit denen man an diese Selbstorganisationsmechanismen herangeht, sind sehr verschieden. Manche kommen aus der Thermodynamik der irreversiblen Prozesse, andere aus der chemischen Kinetik, wie etwa die Arbeiten von Manfred Eigen; andere wiederum greifen auf die Informationstheorie zurück. Interessant ist, dass sich eine ermutigende, wenn auch nicht vollkommene Konvergenz der über die Anwendung ganz verschiedener Methoden erreichten Ergebnisse feststellen lässt. Eine erste Etappe bestand in der Erkenntnis, dass der Begriff der Selbstorganisation streng genommen widersprüchlich ist, wenn man die Organisation als das Funktionsgesetz eines Systems ansieht. Dieses kann sich nicht infolge eines nur internen Determinismus ändern, da dann dieser Determinismus sein - konstantes - Funktionsgesetz bildet.

4 Wie Ashby gezeigt hat, kann die funktionale Organisation nur unter dem Einfluss der Umwelt variieren. Streng genommen kann es also keine Selbstorganisation geben. Doch können die Wirkungen der Umwelt nun in zwei verschiedenen Formen in Erscheinung treten: Entweder handelt es sich um einen programmierten Effekt, das heißt um ein von außen geliefertes Programm (und unter diesen Bedingungen gibt es keinerlei Grund, eine eventuelle Selbstorganisation anzuführen); oder die Umwelteinflüsse sind 
im Gegenteil etwas rein Zufälliges. In diesem Fall kann man durchaus von Selbstorganisation sprechen; um scheinbare Selbstorganisation freilich, denn es existieren ja Umwelteinflüsse, und dennoch Selbstorganisation, weil diese Wirkungen der Umwelt nur zufällig zustande kommen. Die sich nun stellende Frage lautet folgendermaßen: Ist es möglich, sich Systeme von der Art vorzustellen, dass sie auf die zufälligen Effekte der Umwelt reagieren, und zwar so, dass sie ihre Fähigkeiten, auf neue Stimuli zu antworten, also ihre Regulationsfähigkeiten ausbauen? Ashby hat gezeigt, dass, je mehr man die Varietät oder die Heterogenität eines Systems ausweitet, das System sich im Prinzip a priori als umso leistungsfähiger hinsichtlich seiner Regulationsmöglichkeiten, also der Autonomie im Verhältnis zu den zufallsbedingten Störungen der Umwelt erweist.

5 Es ist heute möglich, sich Systeme vorzustellen, die die Wirkungen des Zufalls gewissermaßen integrieren können, was nicht nur heißt, dass sie eine sehr hohe Zuverlässigkeit aufweisen, sondern dass sie dieses Zufällige überdies noch zur Steigerung ihrer eigenen Leistungen nutzen können. Notwendige Bedingungen hierfür sind einerseits eine hohe initiale Redundanz, eine sowohl strukturelle wie auch funktionale Redundanz (strukturelle Redundanz heißt, dass sich die Komponenten des Netzwerks in hoher Zahl wiederholen; funktionale Redundanz bedeutet, dass dieselbe logische Funktion im Netzwerk nicht nur an einem, sondern an verschiedenen Orten ausgeübt wird, die sich so wechselseitig kontrollieren können), andererseits eine hohe Verlässlichkeit mit einer Beziehung zwischen Redundanz und Verlässlichkeit, die jedoch keine Identitätsbeziehung darstellt.

6 Dadurch vermag ein System auf zufällige Einflüsse zu reagieren, indem es sieht, dass seine Redundanz, also seine Verlässlichkeit zurückgeht; sind seine Redundanz und seine Verlässlichkeit hoch, wird es zumindest für eine gewisse Zeit weiterfunktionieren und dies mit einer größeren Varietät sowie einer größeren Heterogenität, die nur die Folge seiner Redundanzverminderung sind, und zwar derart, dass es zu höheren Regulierungsleistungen imstande wäre. Es handelt sich hier um das Order from noisePrinzip, von dem ich bereits gesprochen habe.

7 Sucht man nun diese Gedanken auf eine Theorie des Lernens auszuweiten, gelangt man $\mathrm{zu}$ einer bestimmten Anzahl von Eigenschaften dessen, was man als ein in einem Lernprozess agierendes selbstorganisiertes System ansehen kann. Zunächst muss man zwischen direktivem und nicht-direktivem Lernen unterscheiden. Das erste ist ein Lernen mit einem Lehrer, der einem sagt, was man lernen muss. Beim nicht-direktiven Lernen schafft ein System, das in eine für es neue Umwelt gestellt ist, gewissermaßen in dieser unbekannten Umwelt die patterns, die zu erkennen es sich sodann selbst konditioniert. Diese Mechanismen sind des Weiteren interessant, insofern sie nicht einfach rein phänomenologische Beschreibungen darstellen, sondern in Maschinen reproduziert sind. Ich möchte besonders an den prinzipiellen Unterschied in der Funktionsweise einer Maschine vom Typ »Perceptron« - eine Maschine, die Formen erkennen kann, allerdings mit einem Lehrer (man braucht einen Experimentator, der die Funktionsparameter im Zuge des Lernprozesses an der Maschine selbst einstellt) und einer Maschine vom Typ »Informon«, die Uttley entwickelt hat, erinnern. Es handelt sich dabei um ein derart modifiziertes Perceptron, dass es die Maschine selbst ist, die, indem sie die bedingten Wahrscheinlichkeiten bei den verschiedenen Stimuli, die auf sie wirken, errechnet, gewissermaßen die Häufigkeits-patterns im Anschluss an 
diese Berechnungen fabriziert und dann mehr oder weniger diese patterns in ihrer Umwelt erkennt.

Beim nicht-direktiven Lernen lassen sich zwei Eigenschaften, Folgen des Order from noise-Prinzips, ausmachen: Die erste besteht darin, dass der Lernprozess sich als eine Schöpfung von patterns durch Redundanzminderung begreifen lässt, wobei Spezifizierungen von besonderen patterns andere ausschließen. Doch was nimmt zu und was vermindert sich beim Lernvorgang? Ich glaube, dass man zumindest teilweise eine Antwort darauf bekommt, wenn man sagt, dass es sich bei dem, was beim Lernen zunimmt, um die Differenzierung handelt, das heißt, die Spezifizierung von erlernten patterns, und das impliziert in der Folge eine Zunahme der Varietät, der Heterogenität; im Gegenzug ist das, was sich vermindert, die Redundanz des gesamten Systems, also dessen Undifferenziertheit. Insbesondere könnte man darin eine Erklärung für die Mechanismen der Finalität des Traums und des Schlafs finden, von denen ich bereits gesprochen habe. Michel Jouvet hat uns darüber aufgeklärt, dass es während der paradoxen Schlafphase zu einer Synchronisierung der gesamten Hirnzellen kommt. Vielleicht können wir das als eine Rückkehr zu einem Zustand der extrem hohen initialen Redundanz, d. h. zur Undifferenziertheit erklären. Es scheint ganz so, als ob es ein Lernpotential gebe, das sich durch Redundanz messen ließe. Dieses Potential verringert sich nach und nach im Zuge des Lernprozesses. Und in der Folge wird es notwendig, dass man sich mit Redundanz wiederauflädt, um den Lernprozess wiederaufnehmen und fortführen zu können. Ein zweiter Aspekte des Order from noisePrinzips bei den Mechanismen nicht-direktiven Lernens besteht darin, dass die patterns, sind sie einmal geschaffen, mit den neuen Stimuli abgeglichen oder - genauer - auf sie projiziert und angewendet werden. In dem Maße wie die patterns und neuen Stimuli koinzidieren können, spricht man davon, dass man neue patterns in der Umwelt »erkennt«. Doch in dem Maße, wie sie sich als wirklich neu erweisen, kann diese Koinzidenz nur annäherungsweise sein. Es besteht eine Ambiguität bei dieser Anwendung, bei dieser Projektion der patterns auf die neuen Stimuli, und diese Ambiguität spielt eine positive Rolle, insoweit sie eine Rückkoppelung auf die patterns, d.h. eine Modifikation der anfänglichen patterns zur Folge hat. Diese modifizierten patterns werden in der Folge erneut auf die neuen Stimuli projiziert und so weiter. Man kann sich diese Mechanismen nicht-direktiven Lernens auch durch eine Art Kommen und Gehen von patterns vorstellen, die geschaffen und dann auf zufällige Stimuli projiziert werden, und solchen, die in dem Maße, wie sie nicht exakt mit den Erstgenannten koinzidieren können, dann die Klasse von patterns modifizieren, die als Referenz dient, usw. Anders gesagt sieht es ganz so aus, als ob unser kognitiver Apparat eine Art Apparat wäre, der Ordnung ausgehend vom Rauschen schafft.

\section{BIBLIOGRAPHIE}

Changeux, Jean-Pierre / Danchin, Antoine (1974): »Apprendre par stabilisation sélective de synapses en développement«, in: L'unitél'homme, Paris: Le Seuil, S. 320-350. 
Eigen, Manfred (1971): „Selforganization of matter and the evolution of biological macromolecules«, Die Naturwissenschaften, 58 (10), S. 465-523.

\section{NOTES}

1. Changeux / Danchin (1974), S. 320.

2. Vgl. den ersten Teil, Kapitel 2, Diskussion, S. 76 [L'unité l'homme, Paris: Le Seuil, S. 76, NDLR].

\section{INDEX}

Schlüsselwörter : Order from noise-Prinzip, Selbstorganisation, Redundanz, System, Programme, patterns

Mots-clés : principe d'ordre à partir du bruit, auto-organisation, redondance, système, programme, patterns

\section{AUTEURS}

\section{HENRI ATLAN}

Henri Atlan, Arzt und Philosoph, ist emeritierter Professor für Biophysik. Nähere Informationen finden Sie hier. 